\title{
Evaluación nutricional de adultos mayores con el Mini Nutritional Assessment: MNA
}

\author{
Nutritional assessment of elderly people with the Mini Nutritional Assessment: MNA
}

\author{
Gloria Cecilia Deossa-Restrepo 1*, Luis Fernando Restrepo-Betancur², John Edinson Velásquez-Vargas³, \\ Daniela Varela-Álvarez ${ }^{4}$
}

1 Universidad de Antioquia, Profesora Grupo Académico Nutrición en el Proceso Vital Humano de la Escuela de Nutrición y Dietética. Medellín, Colombia. e-mail: deossa.gloria@gmail.com

2 Universidad de Antioquia, Facultad de Ciencias Agrarias, Escuela de producción agropecuaria, profesor titular diseño experimental. Grupo Giser. Medellín, Colombia. e-mail: frbstatistical@gmail.com

3 Nutricionista Dietista. Universidad de Antioquia, Escuela de Nutrición y Dietética. Medellín, Colombia. e-mail: johneve911@gmail.com

4 Nutricionista Dietista. Universidad de Antioquia, Escuela de Nutrición y Dietética. Medellín, Colombia e-mail: danivarelaal@gmail.com

Fecha de recepción: Agosto 31 - 2015 Fecha de revisión: Mayo 31 - $2016 \quad$ Fecha de aceptación: Diciembre 12 - 2016

Deossa-Restrepo GC, Restrepo-Betancur LF, Velásquez-Vargas JE, Varela Álvarez D. Evaluación nutricional de adultos mayores con el Mini Nutritional Assessment: MNA. Univ. Salud. 2016;18(3):494-504. DOI: http://dx.doi.org/10.22267/rus.161803.54

\begin{abstract}
Resumen
Introducción: El conocimiento relacionado con los adultos mayores, referente a los hábitos de alimentación, características antropométricas y su estado de salud, es de vital interés para entes gubernamentales y privados interesados en esta población, a fin de diseñar estrategias que permitan elaborar diagnósticos y planes de acción. Objetivo: Evaluar el estado nutricional de un grupo de adultos mayores del corregimiento de Güintar del Municipio de Anzá, Antioquia-Colombia. Materiales y métodos: Se utilizaron los siguientes análisis: multivariado de la varianza (MANOVA) con contraste canónico de tipo ortogonal, de correspondencia múltiple, descriptivo de tipo unidimensional y de frecuencias de tipo unidimensional; para lo cual se analizó la información recopilada mediante encuesta, de acuerdo con los factores de discriminación por sexo. Resultados: Se encontró que las mujeres tenían un Índice de Masa Corporal superior al de los hombres; en cuanto al consumo de alimentos se evidenció que el 66,8\% de los adultos mayores ingieren las tres comidas al día, el 21,5\% incorporan lácteos, legumbres y pescado de manera regular, al 50\% les gustan las frutas y 16,7\% consumen más de tres vasos de agua al día. Conclusión: Cerca de una tercera parte de los adultos mayores evaluados presentaron riesgo de malnutrición (más mujeres que hombres) y más del $10 \%$ se encontraba con malnutrición (más hombres que mujeres), situación que amerita replantear las políticas alimentarias para favorecer un estado nutricional adecuado y por ende la salud de este grupo poblacional.
\end{abstract}

Palabras clave: Adulto mayor; estado nutricional; Índice de Masa Corporal. (Fuente: DeCS, Bireme).

\begin{abstract}
Introduction: The knowledge related to elderly people concerning feeding habits, anthropometric characteristics, and their health status, is of vital interest to governmental and private entities interested in this population, in order to design strategies that allow to develop diagnoses and action plans. Objective: To evaluate the nutritional status of a
\end{abstract}


group of elderly people in the town of Güintar from the municipality of Anza in Antioquia, Colombia. Materials and methods: The following analysis were used: multivariate analysis of variance (MANOVA) with canonical contrast of orthogonal type, multiple correspondence, descriptive of one-dimensional type and frequencies of one-dimensional type, where the information gathered through a survey was analyzed according to the factors of discrimination by sex. Results: It was found that women had a higher Body Mass Index than men. In terms of food consumption, it could be seen that $66.8 \%$ of the elderly people eat three meals per day, $21.5 \%$ incorporate dairy products, legumes and fish on a regular basis, $50 \%$ like fruits and $16.7 \%$ consume more than three glasses of water a day. Conclusion: About one third of the elderly people assessed presented risk of malnutrition (more women than men) and more than $10 \%$ were malnourished (more men than women). This situation requires to rethink food policies to promote adequate nutritional status and; therefore, the health of this population group.

Keywords: Elderly; nutritional status; Body Mass Index. (Source: DeCS, Bireme).

\section{Introducción}

En Colombia se consideran adultos mayores (AM) a mujeres y hombres de 60 años o más (o mayores de 50 años si son poblaciones de riesgo, por ejemplo indigentes o indígenas) ${ }^{1}$.

El envejecimiento es un proceso normal por el cual pasan todos los seres vivos, se desarrolla normalmente en las últimas etapas de la vida y se asocia con una alteración progresiva de las respuestas homeostáticas adaptativas del organismo, provocando cambios en la estructura y función de diferentes órganos y sistemas, lo que desencadena mayor vulnerabilidad ante componentes tóxicos del medio y conlleva a enfermedades; entre los cambios más importantes y de mayor prevalencia en este grupo poblacional se destacan los relacionados con los sistemas digestivo, cardiovascular, renal, nervioso central, óseo, muscular, inmunológico y metabólico ${ }^{2-4}$.

Un aspecto propio del avance de la edad es el desgaste o la pérdida de piezas dentales, lo cual puede llevar a la malnutrición por cambios en el consumo y en los métodos de cocción y preparación de los alimentos ${ }^{2}$. Otro condicionante de la salud en esta población es la situación socioeconómica; muchos AM presentan dificultades para trabajar o dependen económicamente de otros, lo cual puede condicionar la calidad de vida y la adquisición de bienes y servicios. En Colombia existen altas tasas de pobreza; un $51.9 \%$ de la población de escasos recursos se ubica en los niveles 1 y 2 del Sistema de identificación de Potenciales Beneficiarios de
Programas Sociales (SISBEN) ${ }^{3}$. Las deficiencias nutricionales se presentan con frecuencia en los AM y pueden generar implicaciones graves en la salud. La prevalencia de malnutrición en hospitales se estima en un 76\% y hasta en un $85 \%$ en hogares de $\mathrm{AM}^{5,6}$, siendo menor en los $\mathrm{AM}$ de la comunidad, en quienes llega a ser de $15 \%{ }^{7,8}$.

Existen diferentes métodos tanto subjetivos como objetivos para evaluar el estado nutricional (EN) de este grupo poblacional, los cuales permiten contextualizar y relacionar el estado socioeconómico y alimentario con el de salud. Dichos métodos están constituidos por indicadores objetivos y subjetivos; entre los primeros sobresalen los indicadores clínicos, que permiten explorar órganos y sistemas y observar la presencia de síntomas o lesiones cutáneas propias de algunas condiciones de salud o relacionadas con el estado de nutrición del organismo $^{9}$ y los antropométricos, los cuales consisten en la medición de dimensiones corporales (peso, talla, perímetros o circunferencias y pliegues de grasa) para conocer la composición corporal, compararla con unos valores de referencia y complementar la información para realizar un diagnóstico del EN ${ }^{10}$. El uso de algunas medidas antropométricas es de gran importancia al momento de determinar la progresión en la pérdida de masa muscular o grasa; mediante el uso del Índice de Masa Corporal (IMC), la Circunferencia Media de Brazo (CMB) y la Circunferencia de Pantorrilla (CP) se puede determinar la fragilidad cuando estos indicadores se encuentran disminuidos ${ }^{11}$. 
En los AM, estas mediciones permiten conocer la progresión en la pérdida de masa magra o muscular y la ganancia o acumulación de grasa corporal $^{11}$. Los indicadores subjetivos hacen referencia a los antecedentes familiares $y$ personales de enfermedades, cambios en el peso, síntomas digestivos y situación socioeconómica; también incluyen los datos relacionados con la alimentación, los cuales permiten conocer la influencia cultural, los patrones de alimentación y la frecuencia de consumo de alimentos, tamaños de porción consumidos, gustos y rechazos alimentarios, permitiendo así realizar una valoración cualitativa y cuantitativa de la alimentación.

Para valorar el EN se usan tamizajes o escalas de valoración rápida, que permiten identificar pacientes con riesgo y/o signos de desnutrición, entre estas se encuentran el Nutritional Screening Initiative (NSI), el Nutritional Risk Index (NRI), la Valoración Global Subjetiva (VGS), el Mini Nutritional Assessment (MNA) y el Malnutrition Universal Screening Tool (MUST); de dichas herramientas, el MNA es un método de cribado o de evaluación que ha sido validado en diferentes países y se utiliza en hospitalización, cuidados en casa 0 en pacientes ambulatorios, incluye medidas antropométricas, datos de estado general y de alimentación ${ }^{12,13}$; es un cuestionario fácil de diligenciar, no requiere de más de 15 minutos y permite una calificación global del EN.

Actualmente se cuenta con gran cantidad de estudios realizados en diferentes poblaciones, culturas y regiones del mundo que aceptan el MNA como el más utilizado y validado por las características positivas que este tiene y que se mencionaron anteriormente ${ }^{10}$.

Contreras et al. ${ }^{14}$, presentan el estudio en el que se aplicó el MNA, y se obtuvieron resultados muy positivos en cuanto a la facilidad de la aplicación y la importancia en la cantidad de AM detectados con riesgo de malnutrición y con malnutrición (entendida la malnutrición en este caso como el déficit o la desnutrición propiamente dicha), mediante una encuesta realizada casa a casa, lo anterior permite comprender la importancia de la aplicación de este instrumento para conocer el EN de los AM de una manera fácil, sencilla y rápida, sin necesidad de hacer movilizar al encuestado y promoviendo un ambiente más tranquilo para la encuesta, además de la posibilidad de realizarla mientras el AM se encuentra con algún familiar.

En México, se realizó un estudio comparativo del EN mediante el uso del MNA en los AM que vivían en diferentes contextos sociales y de salud; el estudio permitió describir la prevalencia de desnutrición en los AM y su relación con los condicionantes que influyen en el estado nutricional ${ }^{15}$; es interesante mencionar entonces, que a pesar de las diferencias en el contexto de vida, la situación de salud y la situación emocional, el MNA fue aplicado a todos estos AM y se pudo obtener un resultado importante que posibilita el trabajo en pro de mejorar las condiciones de vida de ellos, pero permitiendo focalizar la atención hacia los espacios donde se encuentran más casos de desnutrición. El MNA también se utilizó en estudios en 33 centros sociosanitarios del Servicio Regional de Bienestar Social de la Comunidad de Madrid (España), para evaluar la prevalencia de desnutrición relacionada con la enfermedad de los AM.

Resultados preliminares de estudios que comparan el MNA con otros métodos para evaluar el estado nutricional en adultos hospitalizados, demuestran la capacidad potencial de diagnóstico de la desnutrición con el uso del instrumento en mención, pues no observaron subdiagnósticos al compararlo con otros test, al usarlo en los pacientes con fragilidad y de mayor edad ${ }^{16}$.

Las experiencias descritas anteriormente, respaldan la relevancia clínica y social de los datos obtenidos al emplear esta encuesta, teniendo en cuenta que son pocos los estudios publicados en la población adulta mayor colombiana evaluada con el MNA, que permitan validar su aplicación en esta región; por tanto en el presente estudio se evaluó el EN de un grupo de AM del corregimiento de Güintar, del Municipio de Anzá (Antioquia), por medio de la aplicación del MNA.

\section{Materiales y métodos}

La investigación se llevó a cabo entre los años 2014 y 2015, en el corregimiento de Güintar, zona 
rural del municipio de Anzá, ubicado al occidente del departamento de Antioquia (Colombia), en dicho lugar, se realizó una jornada de atención nutricional al AM ambulatorio, en la cual se aplicó la herramienta MNA, método aceptado para valorar el EN de adultos mayores ambulatorios ${ }^{12}$; dicho instrumento fue desarrollado en 1994 por Guigoz et al. ${ }^{17}$ y está validado en diferentes países y se utiliza en hospitalización, cuidados en casa o en pacientes ambulatorios. Está compuesto por dieciocho ítems, que valoran la condición general (morbilidad, estilos de vida, medicación), antropométrica (peso, estatura, circunferencia del brazo, circunferencia de la pantorrilla), dietética (frecuencia de consumo de algunos grupos de alimentos) y subjetiva (autopercepción de salud y nutrición). El puntaje obtenido en dicha escala, permite clasificar al individuo como bien nutrido ( $\geq 24$ ), a riesgo de malnutrición (riesgo de desnutrición: ${ }^{17-23}$ o con malnutrición desnutrición $<$ 17; de igual forma el MNA tiene una escala de valoración del IMC, que permite asignar una puntuación, así IMC $<19=0 ; 19-21=1 ; 21-23=2$; $\geq 23=3$, siendo el puntaje < de 19 el más alterado y considerando el $\geq 23$ como el mejor ${ }^{17,18}$; el MNA no permite medir exceso de peso u obesidad como tal; al aplicar la prueba de tamizaje se trató al máximo de evitar sesgos de memoria y cuando el AM no recordaba alguna pregunta, se le indagaba al cuidador o acompañante.

El corregimiento de Güintar cuenta con 7 veredas y una población cercana a 600 personas $(100$ AM), las cuales residen en aproximadamente 200 casas $^{19}$. Disponen de un puesto de salud que ofrece a la comunidad los servicios de promoción, prevención y de atención básica; cuenta además con un gerontólogo (profesional que guía y apoya al AM en las diferentes circunstancias que enfrenta durante el envejecimiento) ${ }^{20}$ y con personal de apoyo en la atención en salud y con un centro día (espacios de participación e inclusión para el AM) ${ }^{21}$.

El tipo de estudio fue descriptivo, exploratorio de tipo comparativo entre sexos. Se efectuó con base en una muestra de $80 \mathrm{AM}$, donde se empleó la técnica denominada muestreo aleatorio de proporciones con factor de ajuste por finitud, teniendo en cuenta un error máximo permisible del 5\% y un nivel de confiabilidad del 95\%, el parámetro $\mathrm{P}$ asumió el valor de 0.5 a fin de maximizar el tamaño definitivo de la muestra, se utilizó el modelo binomial para la selección aleatoria de las personas, anotando que el tamaño de la población objetivo estaba conformado por 100 AM. Para llevarlo a cabo se retomaron algunas de las preguntas del MNA ${ }^{17,18}$. Para incluir a los AM en la toma de los datos se tuvieron como criterios que no presentaran enfermedades graves, que pudieran movilizarse por sí mismos, que se les pudieran tomar los datos de peso y talla, al igual que estuvieran en capacidad de responder las preguntas de la encuesta. Los datos fueron tomados por estudiantes de Nutrición y Dietética de último año de formación entrenados en el manejo del instrumento.

Se obtuvieron medidas antropométricas como peso y talla para hallar el índice de masa corporal (IMC); para la medición del peso corporal se utilizó una báscula digital (Tanita HD314) con una capacidad de $150 \mathrm{~kg}$ y $0.1 \mathrm{~kg}$ de sensibilidad, el peso se tomó con ropa liviana y la mínima cantidad de accesorios. La estatura se midió con un estadímetro portátil (marca SECA) con cinta métrica metálica, cuerpo en pasta, pieza fija y escuadra móvil, con una longitud de $220 \mathrm{~cm}$ y una sensibilidad de $0.1 \mathrm{~cm}$.

El análisis estadístico de la información se realizó por medio de la técnica multivariada de la varianza (MANOVA) con contraste canónico ortogonal, determinando la dimensionalidad de la comparación multidimensional, por medio del criterio de máxima verosimilitud observando el mayor valor propio significativo y con base en el mismo se procedió a efectuar un análisis de varianza canónico por medio de la función correspondiente, dicho análisis se realizó con el fin de comparar la divergencia entre géneros para las variables antropométricas. El análisis se complementó por medio de la técnica multivariada de correspondencia múltiple con clasificación jerárquica indexada, pruebas comparativa de proporciones entre sexos método Z. Adicionalmente se realizó estadística descriptiva unidimensional, a fin de establecer la media aritmética, la desviación típica, el coeficiente de variación, el valor máximo y 
mínimo por pregunta, asociado a variables cuantitativas. Se utilizaron los paquetes estadísticos SAS UNIVERSITY y SPAD versión 3.5. La base de datos obtenida, se exportó al programa Excel versión 2010, efectuando un control de calidad sobre el sistema matricial generado y concatenándola con los paquetes estadísticos utilizados.

\section{Consideraciones éticas}

Según el Ministerio de Salud de Colombia, en la Resolución Número 008430, de octubre de 1993, Artículo 11, esta investigación se clasifica con riesgo mínimo, cumplió con todas las consideraciones éticas y fue aprobado por el Comité de Bioética de la Facultad de Odontología de la Universidad de Antioquia, según Acta número 01 de febrero 27 de 2015.

Todos los adultos mayores indagados aceptaron la participación en el estudio de manera voluntaria. El estudio cumplió con los principios éticos del código de Nuremberg de 1947, con el Informe Belmont del 18 de abril de 1979, la Declaración de Helsinki y Resolución 13437, Artículo 1 que corresponde a los derechos de los pacientes, numeral 8, del Ministerio de Salud, de 1991.

\section{Resultados}

En la mayoría de individuos se encontró un EN normal, de acuerdo a la clasificación del MNA (12 puntos o más en la aplicación del cribaje (ítems A-
F). El riesgo de malnutrición fue mayor en las mujeres $37,3 \%$ que en los hombres $30 \%$, presentando diferencia significativa $(\mathrm{p}<0,05)$; sin embargo la malnutrición fue mayor en los hombres $16,7 \%$ con respecto a las mujeres $11,8 \%$, siendo significativa la diferencia $(\mathrm{p}<0,05)$. (Tabla 1).

Tabla 1. Porcentaje de adultos mayores a riesgo o con malnutrición

\begin{tabular}{|c|c|c|c|}
\hline $\begin{array}{l}\text { Clasificación } \\
\text { Nutricional }\end{array}$ & $\begin{array}{c}\text { Masculino } \\
\% \\
\end{array}$ & $\begin{array}{c}\text { Femenino } \\
\%\end{array}$ & $\begin{array}{c}\text { Total } \\
\%\end{array}$ \\
\hline Normal & $53,3 \mathrm{a}$ & $50,9 a$ & 52,1 \\
\hline $\begin{array}{l}\text { Riesgo mal } \\
\text { Nutrición (riesgo } \\
\text { de desnutrición) }\end{array}$ & $30,0 \mathrm{~b}$ & $37,3 \mathrm{a}$ & 33,7 \\
\hline $\begin{array}{l}\text { Mal Nutrición } \\
\text { (desnutrición) }\end{array}$ & $16,7 \mathrm{a}$ & $11,8 \mathrm{~b}$ & 14,3 \\
\hline
\end{tabular}

La edad promedio de los AM del género masculino fue de 67,8 años con una dispersión de 8,9 años, mientras que las mujeres tenían una edad promedio de 65,7 años con una dispersión de 9,4 años. El peso promedio de los hombres fue de 60,3 $\mathrm{kg}$ y el de las mujeres de $60,6 \mathrm{Kg}$. La talla promedio de los AM del género masculino fue de $1,58 \mathrm{~m}$ y de $1,50 \mathrm{~m}$ para las mujeres y fueron estas quienes presentaron un IMC superior al de los hombres. Al efectuar el análisis multivariado de la varianza para las variables antropométricas, se presentó diferencia altamente significativa entre sexos $(\mathrm{p}<0,0001)$. (Tabla 2$)$.

Tabla 2. Análisis descriptivo y de la varianza MANOVA variables antropométricas

\begin{tabular}{|c|c|c|c|c|c|c|c|c|c|c|}
\hline \multirow[t]{2}{*}{ Variable } & \multicolumn{4}{|c|}{ Masculino } & \multicolumn{6}{|c|}{ Femenino } \\
\hline & Media \pm Sd & $\mathbf{C v}$ & Max & Min & \multicolumn{3}{|c|}{ Media \pm Sd } & $\mathrm{Cv}$ & Max & Min \\
\hline Edad & $67,8 \pm 8,9 \mathbf{a}$ & 13,1 & 84,0 & 51,0 & \multicolumn{3}{|c|}{$65,7 \pm 9,4 \mathbf{a}$} & 14,4 & 87,0 & 50,0 \\
\hline Peso & $60,3 \pm 8,5$ a & 14,0 & 74,8 & 45,5 & \multicolumn{3}{|c|}{$60,6 \pm 9,0 \mathbf{a}$} & 14,9 & 79,0 & 42,0 \\
\hline Talla & $1,58 \pm 0,07 \mathbf{a}$ & 4,9 & 1,77 & 1,42 & \multicolumn{3}{|c|}{$1,50 \pm 0,05 \mathbf{b}$} & 3,8 & 1,6 & 1,4 \\
\hline IMC & $24,1 \pm 3,6 \mathbf{b}$ & 15,0 & 33,6 & 18,2 & \multicolumn{3}{|c|}{$26,7 \pm 3,6 \mathbf{a}$} & 13,7 & 14,6 & 18,2 \\
\hline \multicolumn{11}{|c|}{ MANOVA } \\
\hline & \multicolumn{2}{|c|}{ Wilks' Lambda } & \multicolumn{2}{|c|}{0,70168784} & 8,08 & 4 & 76 & $<, 0001$ & & \\
\hline & \multicolumn{2}{|c|}{ Pillai's Trace } & 0,29 & 31216 & 8,08 & 4 & 76 & $<, 0001$ & & \\
\hline & \multirow{2}{*}{\multicolumn{2}{|c|}{$\begin{array}{l}\text { Hotelling-Lawley Trace } \\
\text { Roy'sGreatestRoot }\end{array}$}} & \multicolumn{2}{|c|}{0,42513514} & 8,08 & 4 & 76 & $<, 0001$ & & \\
\hline & & & 0,42 & 13514 & 8,08 & 4 & 76 & $<, 0001$ & & \\
\hline
\end{tabular}


Al indagar a los adultos mayores sobre algunos aspectos relacionados con su salud y hábitos de alimentación, se encontró que el 98,7\% presentaba autonomía en la movilidad, 25,0\% reportó comer menos de lo habitual, 21,3\% había perdido entre uno y tres kilos, 41,2\% había presentado enfermedades agudas o estrés psicológico, 25\% consideraba haber presentado demencia o depresión moderada y $76,3 \%$ tenía un IMC por encima de 23 que es el valor que considera el instrumento MNA como el mejor (Tabla 3).

Tabla 3. Análisis porcentual de los hábitos de alimentación y condición de salud y nutrición de los AM del corregimiento de Güintar (Anzá), Antioquia-Colombia

\begin{tabular}{lr}
\hline Preguntas & \multicolumn{1}{l}{$\%$} \\
\hline Vive independiente en su domicilio & 38,0 \\
Ha comido menos de lo habitual & 33,7 \\
Ha comido igual & 66,3 \\
No sabe si ha perdido peso & 20,0 \\
Ha perdido entre 1 y 3 Kg & 21,3 \\
Ha perdido más de 3 Kg & 8,7 \\
No ha perdido peso & 50,0 \\
Movilidad de la cama al sillón & 1.3 \\
Autonomía en la movilidad & 98,7 \\
Ha tenido enfermedades agudas o estrés & 41,2 \\
psicológico & \\
Ha presentado demencia o depresión grave & 1,3 \\
Ha presentado demencia o depresión & 25,0 \\
moderada & \\
Sin problemas de depresión o de demencia & 73.7 \\
IMC<19 & 2,5 \\
19<IMC<21 & 8,7 \\
IMC & 12,5 \\
\hline & \\
\hline &
\end{tabular}

A los adultos mayores que resultaron con puntaje en el cribado de 11 o menos, es decir que tendrían una posible malnutrición (desnutrición 47,5\% n= 38 ), se les continuó realizando la evaluación del cuestionario, encontrando que 38\% vivía de forma independiente, $25,5 \%$ tomaba más de tres medicamentos por día, 19\% reportó presentar úlceras en la piel o en alguna parte de su cuerpo, $66,8 \%$ consumía tres comidas al día, 21,5\% consumía lácteos, legumbres y pescado de manera regular, 50\% consumía frutas, 16,7\% consumía más de cinco vasos de agua al día, $83,3 \%$ se alimentaba solo, $35,7 \%$ se auto consideraba con malnutrición moderada y 14,3\% consideraba que su estado de salud estaba peor cada día (Tabla 4).

Tabla 4. Distribución porcentual de los hábitos de vida, alimentación y condición de salud de los AM del corregimiento de Güintar (Anzá), AntioquiaColombia

\begin{tabular}{ll}
\hline Preguntas & $\%$
\end{tabular}

Toma más de tres medicamentos al día $\%$

Presenta úlceras o lesiones cutáneas 19,0

Realiza una comida completa al día

Realiza dos comidas completas al día

28,5

Realiza tres comidas completas al día

66,8

Consume: lácteos, legumbres, pescado

21,5

Consume frutas

50,0

Consume menos de tres vasos de agua

50,0

Consume entre 3 y 5 vasos de agua

33,3

Consume más de 5 vasos de agua

16,7

Necesita ayuda para alimentarse

Se alimenta solo sin dificultad

83,3

Se alimenta solo con dificultad

14,4

El paciente se considera a sí mismo con

malnutrición grave

El paciente se considera con malnutrición $\quad 35,7$ moderada

El paciente se considera sin malnutrición $\quad 62,0$

Como encuentra el paciente su estado de salud $\quad 14,3$ (peor)

Como encuentra el paciente su estado de salud 23,8 (igual)

Como encuentra el paciente su estado de salud 61,9

(mejor) 
Al realizar el análisis factorial de correspondencia múltiple, se establecieron las siguientes similitudes entre el conjunto de variables de carácter cualitativo: los AM que han consumido menos cantidad de comidas presentan correlación positiva con un IMC inferior a 19, ellos no saben si han perdido peso y los que afirman haber perdido más de tres kilos, también reportaron presentar alguna enfermedad aguda o estrés psicológico. De manera adicional en dicho plano se observa que no existen diferencias entre géneros y una correlación positiva entre los AM que han comido igual con la presencia de autonomía en los movimientos, con IMC entre 19 y 23, con ausencia de enfermedades agudas o de estrés psicológico; aunque algunos reportan haber perdido entre uno y tres kilos (Figura 1).

Figura 1. Plano factorial enfermedades $y$ comportamiento del anciano

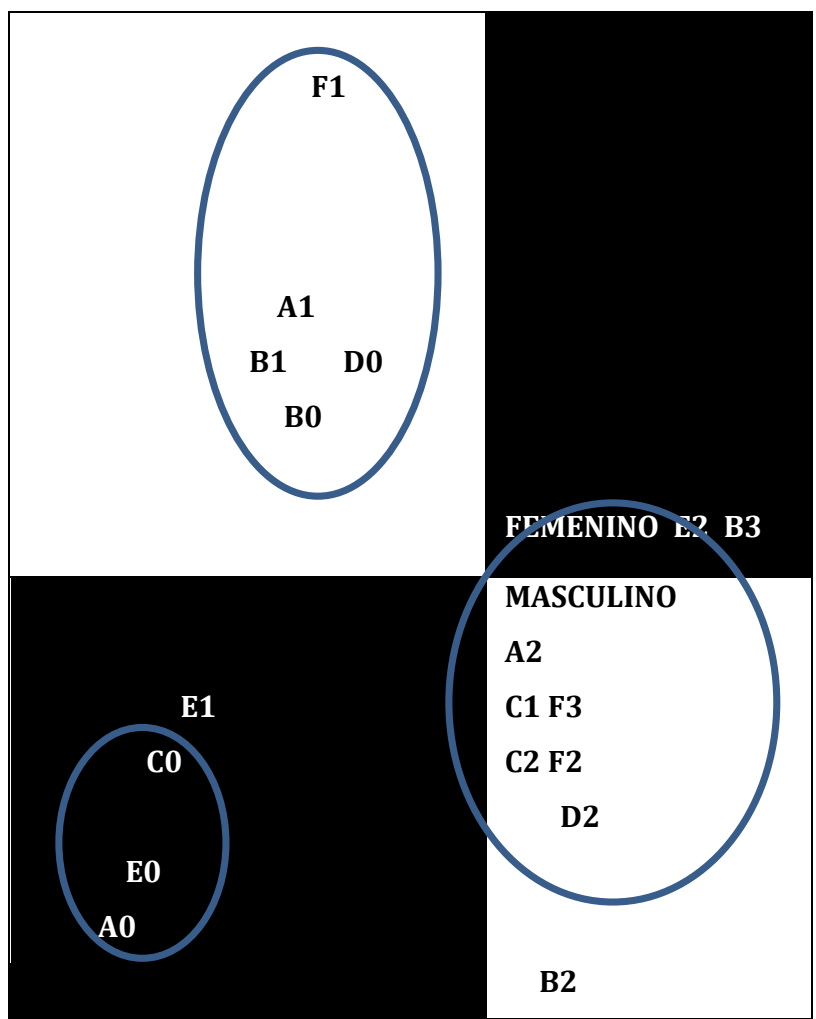

$\mathbf{A 0}=$ Ha comió mucho menos; $\mathbf{A 1}=$ ha comido menos; $\mathbf{A 2}=$ Ha comido igual; $\mathbf{B O}=$ Pérdida de peso $>3 \mathrm{Kg}, \mathbf{B 1}=$ No lo sé, $\mathbf{B} 2=$ Perdida entre 1 y $3 \mathrm{Kg} ; \mathbf{B 3}=$ No ha perdido peso; $\mathbf{C 0}=$ De la cama al sillón, $\mathbf{C 1}=$ Autonomía en el interior; $\mathbf{C 2}=$ Sale del domicilio; $\mathbf{D 0}=$ Ha tenido enfermedad aguda o estrés psicológico; D2 $=$ No ha tenido enfermedad aguda o estrés psicológico; E0= Demencia o depresión grave; E1 = Demencia o depresión moderada; E2 = Sin problemas; F0= IMC $<19 ; \mathbf{F 1}=19<\mathrm{IMC}<21 ; \mathbf{F} 2=21<\mathrm{IMC}<23 ; \mathbf{F 3}=\mathrm{IMC}>23$.
En la Figura 2, se aprecian las siguientes similitudes: los AM que consumen entre tres a cinco vasos de agua por día se relacionan con el consumo de más de tres medicamentos por día y con la incorporación de dos alimentos entre (pescado, lácteos y legumbres) en su comida. Los AM con un consumo de tres alimentos al día se caracterizaron por incorporar frutas en su comida, sentirse igual a otras personas de su edad, vivir de manera independiente y no saben si están malnutridos; es de anotar que las mujeres tienden a consumir con mayor frecuencia las tres comidas al día con respecto a los hombres.

Figura 2. Plano factorial relacionado con hábitos (alimenticios, medicamentos)

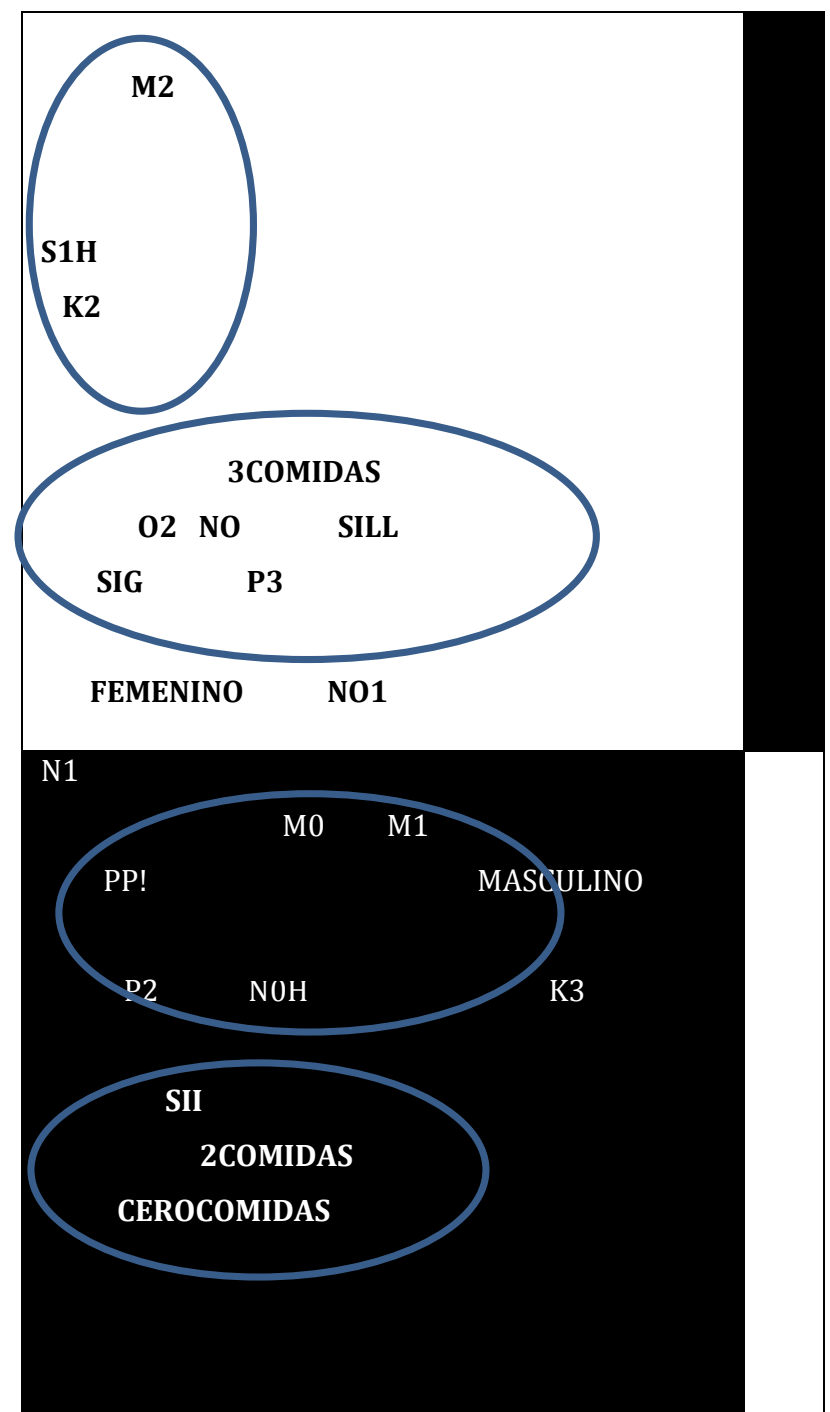

$\mathbf{S I G}=\mathrm{El}$ paciente vive de manera independiente; $\mathbf{N O G}=\mathrm{El}$ paciente no vive independientemente; $\mathbf{S I H}=$ Toma más de tres medicamentos al día; $\mathbf{N O H = ~ N o ~ t o m a ~ m a ́ s ~ d e ~ t r e s ~ m e d i c a m e n t o s ~ a l ~ d i ́ a ; ~} \mathbf{S I I}=$ Posee 
úlceras o lesiones cutáneas; NOL=No posee lesiones o ulceras cutáneas; $\mathbf{K}=$ Consume lácteos, legumbres, pescado. K0= Cero o 1 alternativa de $\mathrm{k} ; \mathbf{K} \mathbf{1}=\mathbf{2}$ alternativas de $\mathrm{k}, \mathbf{K} \mathbf{2}=$ Las tres alternativas de $\mathrm{k}$; $\mathbf{S I L L}=\mathrm{Si}$ consume frutas o verduras 2 veces al día; $\mathbf{N O L L}=$ No consume frutas o verduras 2 veces al día; M0= Toma menos de 3 vasos de agua, K1= Entre 3 y 5 vasos de agua, K2= Más de 5 vasos de agua; N1=Necesita ayuda para alimentarse; N2= Se alimenta solo con dificultad; 01 = Malnutrición grave; $\mathbf{0 2}=$ Malnutrición moderada; $\mathbf{P}=$ Peor se encuentra paciente con respecto a personas de su misma edad; PI=No lo sabe; P2=Igual; P3=Mejor.

\section{Discusión}

Diversos estudios refieren la utilización del MNA como método de cribado para evaluar el EN de la población de adultos mayores, por ser una herramienta fácil y rápida de usar, útil en pacientes hospitalizados, institucionalizados o ambulatorios y que permite tomar decisiones para mejorar el estado de salud $\mathrm{d}^{22-24}$.

Los hallazgos de este estudio son similares a los reportados en otros países de Latinoamérica; en un estudio realizado en el año 2012 en la provincia de Tungurahua (Ecuador), que tenía como objetivo evaluar el EN por medio del MNA en $90 \mathrm{AM}$ no institucionalizados, se encontró que $43,3 \%$ presentaba un EN normal, el 27,8\% riesgo de malnutrición y 28,9\% malnutrición 25 ; nuestros hallazgos indican que la mayoría de los AM evaluados presentaron un EN normal; no obstante el $30 \%$ de los AM pertenecientes al género masculino presentó riesgo de malnutrición, pero fueron las mujeres quienes presentaron diagnóstico elevado con un 37,7\%.

En el año 2010, se evaluó por medio del MNA a 157 AM (93 hombres y 64 mujeres) mexicanos que asistían a consulta de medicina familiar, encontrando en promedio un IMC de 26.9, una talla de 1,57 mt y un peso de $66,7 \mathrm{~kg}$; en el presente estudio el IMC promedio fue de 24,1 $\mathrm{kg} / \mathrm{mt}^{2}$ en los hombres y de $26,7 \mathrm{~kg} / \mathrm{mt}^{2}$ en mujeres, mostrando que ellas presentaban un IMC mayor, cercano al valor reportado en el estudio en México; además se relaciona con hallazgos de otros estudios, en los cuales hay una relación positiva entre la obesidad, la edad y el sexo femenino ${ }^{26}$.

En una investigación realizada con 660 AM en España, en donde se quería valorar el EN mediante la aplicación del MNA, se encontró que 48,3\% tomaba más de 3 medicamentos al día; 9,7\% presentaba úlceras cutáneas; 23,9\% consumía entre 3 y 5 vasos de agua u otros líquidos al día; el 75,4\% consumía más de 5 vasos al día y el $100 \%$ se alimentaba solo sin dificultad ${ }^{27}$. Al comparar estos datos con los resultados de este estudio, se encuentra que es menor la proporción de AM que toma más 3 medicamentos al día $(25,5 \%)$, pero mayor el número que reportó úlceras cutáneas (19,9\%); solo el 16,7\% ingerían más de 5 vasos de agua al día, muy por debajo de los hallazgos del estudio de España, es importante este hallazgo, pues en esta etapa de la vida se pierde el mecanismo de sensación de sed y disminuye rápidamente la masa muscular, compuesta entre otros por proteínas y agua 28 ; el $83,3 \%$ de los AM de estudio se alimentaba solo y sin dificultad, dato inferior al reportado en España, en el cual 100\% de los evaluados reportó autonomía en la alimentación.

Con respecto a la percepción que los AM tienen de su estado nutricional, se encontró que 2,3\% se considera con malnutrición grave, $35,7 \%$ con malnutrición moderada y $62 \%$ sin malnutrición; en una investigación realizada en la provincia de Ourense, España con 728 AM, se reportó que $0.4 \%$ se consideraba con malnutrición grave, 16,1\% con malnutrición moderada y $83,5 \%$ sin malnutrición ${ }^{29}$, notando como los AM sienten más alterado su estado nutricional que los del estudio de España. Las alteraciones psicológicas afectan a los AM y en especial a los habitantes de poblaciones rurales de escasos recursos y de países en vía de desarrollo; según la teoría de los determinantes sociales, el hecho de vivir en un país en vía de desarrollo, condiciona con más fuerza el desarrollo de enfermedades o situaciones socioculturales, con respecto a los países desarrollados ${ }^{30}$.

En un estudio realizado en España, en el que se evaluó por medio del MNA a $22.007 \mathrm{AM}$, se encontró que $27,9 \%$ presentó enfermedades agudas o estrés psicológico; $21,7 \%$ depresión moderada; $2,4 \%$ depresión grave y $75,9 \%$ reportó no haber presentado depresión ${ }^{31}$; al evaluar la población AM de Güintar, se encontró que 41,2\% reportaron haber sufrido enfermedades agudas 0 
estrés psicológico, 25\% depresión moderada; $1,3 \%$ depresión grave y $73,7 \%$ dicen que no han presentado depresión (Tabla 3), valores muy similares a los reportados en España, excepto en lo concerniente al padecimiento de enfermedades agudas o estrés psicológico, el cual fue más prevalente en la población de Güintar y que puede estar relacionado con los condicionantes psicosociales y al hecho de pertenecer a una población rural, en su mayoría de escasos recursos, relación que se encuentra también reportada en diferentes lugares del mundo ${ }^{32}$, lo cual explica que existe una correspondencia negativa entre el estado socioeconómico y la aparición de enfermedades mentales relacionadas con el estrés.

En Colombia, en el año 2011 se realizó un estudio donde se aplicó también el MNA a 80 AM de la zona urbana de Bogotá, para determinar los factores de riesgo asociados al EN y validar la información obtenida a partir de las respuestas del informante, cuidador o familiar, encontrando que entre los factores de riesgo más prevalentes asociados al EN fueron los relacionados con los datos dietéticos, se observó que el $86 \%$ de la población no consumía carne, pescado o aves diariamente; $84 \%$ no consumía frutas o verduras dos veces al día y $70 \%$ consumía únicamente dos comidas al día ${ }^{33}$. En el presente estudio, el 66,8\% reportó consumir las tres comidas al día; $21,5 \%$ incorporan lácteos, leguminosas y pescado de manera regular; se evidencia el bajo consumo de alimentos fuentes de proteína de origen animal, nutriente importante en esta etapa de la vida, en la cual se presenta más riesgo de enfermedades infecciosas y una pérdida acelerada de la masa muscular 28; además los alimentos fuentes de proteína, lo son también de otros micronutrientes importantes para el mantenimiento de la salud, como es el caso del zinc, el selenio, las vitaminas B6 y B12, todos importantes para un envejecimiento saludable ${ }^{34}$.

Investigaciones de corte cualitativo efectuadas en la ciudad de Medellín como la realizada por Franco y Arboleda, en 2010, con el objetivo de "interpretar los aspectos socioculturales y técnico-nutricionales de la alimentación de un grupo de AM del centro gerontológico Colonia de
Belencito", evidenció que para los AM es muy importante la alimentación, ya que según ellos es la que les da la energía para las labores del día, igualmente se encontró que los AM reconocen unos alimentos que son sanadores y que proveen al cuerpo de ciertos beneficios para la salud, entre los que se destacan las frutas y verduras ${ }^{35}$. En este estudio no se indagó por la percepción del papel de los alimentos para la salud y el bienestar físico, sin embargo, se pudo observar que los AM le prestan mucha importancia al consumo de alimentos durante el día y que se puede evidenciar, dado que la mayoría de los adultos consumen al menos 3 comidas al día, e incluyen las frutas, verduras y lácteos, grupos de alimentos que hacen parte de una alimentación saludable (Tabla 4).

El 50\% de los AM de esta investigación incluye las frutas en alguna de las comidas del día, hallazgo que amerita resaltarse, puesto que en la Encuesta Nacional de la Situación Nutricional de Colombia, ENSIN 201036, se evidenció un bajo consumo de este grupo de alimentos por parte la población de $31-64$ años, ya que solo $38 \%$ de dicho grupo etario reportó consumirlas; estos hallazgos evidencian que en el grupo de AM evaluados, existe más conciencia sobre el consumo de estos alimentos, los cuales aportan gran cantidad de nutrientes que favorecen la salud; además la población vive en una zona rural, de clima cálido y esto permite un mayor acceso a estos alimentos.

Entre los principales hallazgos del estudio sobre envejecimiento y vejez en Colombia, realizado en el año 2013, se encontró "escasa seguridad económica en la vejez", "revelan percepciones menos favorables sobre su salud y mayores limitaciones funcionales, así como disminución en la satisfacción con la familia" 37 ; en este estudio se pudo evidenciar que la mayoría de los AM vive independiente, sin embargo las condiciones económicas son precarias, similares a las del resto del país. En cuanto al consumo de medicamentos, se observó que la mayoría consume más de 3 medicamentos al día, lo cual se relaciona con el deterioro de la salud propio de los AM y de los procesos de envejecimiento. 
Entre las limitaciones del estudio se encuentran la falta de la toma de medidas antropométricas como circunferencia de pantorrilla, perímetro abdominal y perímetro braquial, para realizar un mejor análisis de las variables estudiadas.

\section{Conclusión}

Cerca de la mitad de los adultos mayores evaluados presentaron un estado nutricional normal, mientras que el resto presentó riesgo de malnutrición o malnutrición como tal determinada por el MNA, hallazgos importantes para definir y re-direccionar las políticas de alimentación y nutrición dirigidas a este grupo poblacional y de este modo contribuir al mantenimiento de la salud y a una mejor calidad de vida de estas personas, que han logrado llegar a esta etapa de la vida y que merecen vivirla de manera saludable y digna.

\section{Agradecimientos}

A la Dirección de la Escuela de Nutrición y Dietética de la Universidad de Antioquia por el apoyo económico para la realización de este estudio, a los estudiantes de Nutrición y Dietética que participaron en la toma de los datos, por el diligenciamiento de la encuesta y participación activa y al presbítero de la localidad por toda la inmensa colaboración para citar a los AM.

Conflicto de intereses: Ninguno declarado por los autores.

\section{Referencias}

1. República de Colombia. Ministerio de la Protección Social. Política Nacional de Envejecimiento y Vejez, 2007-2019.2007

2. Gobierno federal de los estados unidos mexicanos. Guía práctica clínica: Diagnóstico y manejo de los problemas bucales en el adulto mayor. [Internet]. [Consultado el 12 de Mayo 2015]. Disponible en: http://www.cenetec.salud.gob.mx/descargas/gpc/Cata logoMaestro/583_GPC_Problemasbucalesenadultomay or/583GRR.pdf

3. Gobernación de Antioquia. Plan de desarrollo. adulto mayor. [Internet]. [Consultado el 17 de febrero 2015]. Disponible

en:

http://www.antioquia.gov.co/index.php/plan-dedesarrollo/2512-adulto-mayor
4. Saleche F, Jara L, Michea L. Cambios Fisiológicos asociados al envejecimiento. Rev. Med. Clin. Condes. 2012; 23(1) 19-29.

5. Argente $M$, et al. prevalencia de desnutrición en una unidad de media y larga estancia hospitalaria. Nutr Hosp. 2015;31(2):900-907

6. Serrano-Urrea R, Garcia-Meseguer M. Malnutrition in an elderly population without cognitive impairment living in nursing homes in Spain: study of prevalence using the Mini Nutritional Assessment test. Gerontology. 2013; 59(6): 490-498.

7. Martone A, Onder G, Vetrano D, Ortolani E, Tosato M, Marzetti E, et al. (2013). Anorexia of aging: a modifiable risk factor for frailty. Nutrients. 2013;5 (10): 41264133.

8. Morley J. Undernutrition in older adults. Family Practice 2012; 29:i89-i93

9. Cañete R, Cifuentes V. Valoración del estado nutricional [internet]. [Consultado el 17 de febrero 2014]. Disponible

en:http://www.seep.es/privado/documentos/publicac iones/2000TCA/Cap01.pdf

10. Varela LF. Nutrición en el adulto mayor. Rev Med Hered. 2013; 24:183-185.

11. Babiarczyk B, Tubiarz A. Body Mass Index in Elderly People - do the reference ranges matter? Prog Health Sci. 2012; 2(1): 58-67

12. Cereda E. Mini nutritional assessment. Current Opinion in Clinical Nutrition \& Metabolic Care. 2012; 15(1):2941.

13. Skipper A, Ferguson M, Thompson K, Porcari J. Nutrition screening tools: An analysis of the evidence. JPEN Nutr. 2012;36:292-298

14. Contreras AL, Mayo GVA, Romaní DA, et al. Malnutrición del adulto mayor y factores asociados en el distrito de Masma Chicche, Junín, Perú. Rev Med Hered. 2013; 24:186-191.

15. Osuna IA, Verdugo S, Leal G, Osuna I. Estado nutricional en adultos mayores mexicanos: estudio comparativo entre grupos con distinta asistencia social. Rev Esp Nutr Hum Diet. 2015; 19(1): 12 - 20.

16. Adrian Slee, Deborah Birch, David Stokoe. A comparison of the malnutrition screening tools, MUST, MNA and bioelectrical impedance assessment in frail older hospital patients. Clin Nut. 2015; 34(2):296-301

17. Guigoz Y, Vellas B, Garry PJ. Assessing the nutritional status of the elderly: The Mini Nutritional Assessment as part of the geriatric evaluation. Nutr Rev [Internet]. 1996 [citado 2015 Sep 29]. 54(1 Pt 2):S59-65. Disponible http://www.ncbi.nlm.nih.gov/pubmed/8919685

18. Vellas B, Guigoz Y, Garry PJ, Nourhashemi F, Bennahum $D$, Lauque $\mathrm{S}$, et al. The Mini Nutritional Assessment (MNA) and its use in grading the nutritional state of elderly patients. Nutrition [Internet]. 1999 [citado 2015 Abr 16]. 15(2):116-22. Disponible en: http://www.ncbi.nlm.nih.gov/pubmed/9990575

19. Güintar. Corregimiento de Güintar. [internet]. [Consultado el 17 de febrero 2015]. Disponible en: http://www.guintar.com.co/p/corregimiento-deguintar-el.html 
20. Universidad Autónoma del Estado de Hidalgo. Licenciatura en Gerontología. [internet]. [Consultado el 05 de julio 2015]. Disponible en: http://www.uaeh.edu.mx/campus/icsa/gerontologia/l ic_gerontologia.pdf

21. República de Colombia. Ministerio de la Protección Social. Ley 1315 de 2009, establece las condiciones mínimas que dignifiquen la estadía de los adultos mayores en los centros de protección, centros de día e instituciones de atención. Publicada en el Diario Oficial 47.409 de julio 13 de 2009.

22. Salvà Casanovas A. The Mini Nutritional Assessment. Twenty years contributing to nutritional assessment. Rev Esp Geriatr Gerontol. 2012;47(6):245-246

23. Zwenger Y, Salinas S,Cicchitti A, Pool MC y Russo A. Herramientas de screening nutricional. Buenos Aires: Asociación Argentina de Nutrición Enteral y Parenteral; 2011

24. Slee A, Birch D, Stokoe D. A comparison of the malnutrition screening tools, MUST, MNA and bioelectrical impedance assessment in frail older hospital patients. Clinical Nutrition. 2015; 34 (2): 296301

25. Palacios S. Evaluación del estado nutricional mediante el MNA (Mini Nutritional Assessment) en relación a factores sociales y demográficos en adultos mayores no institucionalizados de la parroquia Rumipamba, provincia de Tungurahua. 2011. [Para Optar el Titulo de: Nutricionista Dietista]. Riobamba-Ecuador: Pontificia Universidad Javeriana. 2012

26. Calderón Reyes ME, Ibarra Ramírez F, García J, Gómez Alonso C, Rodríguez-Orozco AR. Evaluación nutricional comparada del adulto mayor en consultas de medicina familiar. Nutr. Hosp.2010; 25(4): 669-675.

27. Montejano Lozoya R, Ferrer Diego R, Clemente Marín G, Martínez-Alzamora N. Estudio del riesgo nutricional en adultos mayores autónomos no institucionalizados. Nutr. Hosp. [revista en la Internet]. 2013; 28(5): 14381446.

28. Alfaro L. Evaluación nutricional en adultos mayores en una Unidad de Medicina Familiar. 2010. [Para Optar el Titulo de: Especialista en Medicina Familiar]. QuerétaroMéxico: Universidad Autónoma de Querétaro. 2010

29. De la Montaña J, Areal C, Míguez M. Evaluación del riesgo nutricional mediante el MNA en una población anciana no institucionalizada. Arch. Latinoam. Nutr. 2009: 59(4); 390-395

30. Organizaciòn mundial de la salud. Determinantes sociales en salud. [Internet]. [2015 Agosto 22]Disponible http://www.who.int/social_determinants/es/

31. Cuervo M, García A, Asorena D, Sánchez-Villegas A, Martínez-González MA, Astiasarán A, et al. Nutritional assessment interpretation on 22007 Spanish community-dwelling elders through the Mini Nutritional Assessment test. Public Health Nutrition. 2008:12(1); 82-90.

32. Ortiz-Hernández L, López-Moreno S, Borges G. Desigualdad socioeconómica y salud mental: revisión de la literatura latinoamericana Socioeconomic inequality and mental health: a Latin American literature review. Cad. saúde pública. 2007: 23(6); 1255-1272.

33. González JL. Evaluación del estado nutricional de un grupo de adultos mayores pertenecientes al plan nueva sonrisa dispensario Santa Francisca Romana. [Para Optar el Titulo de: Nutricionista Dietista]. Bogotá: Pontificia Universidad Javeriana. 2011.

34. Martínez Roldán C, Veiga Herreros P, Cobo Sanz J, Carbajal Azcona A. Evaluación del estado nutricional de un grupo de adultos mayores de 50 años mediante parámetros dietéticos y de composición corporal. Nutr. Hosp. 2011; 26(5): 1081-1090.

35. Franco Giraldo FA, Arboleda Montoya LM. Aspectos socioculturales y técnico-nutricionales en la alimentación de un grupo de adultos mayores del centro gerontológico Colonia de Belencito de MedellínColombia. Perspect Nutr Humana. 2010; 12(1): 61-74.

36. Instituto Colombiano de Bienestar Familiar, Ministerio de la Protección Social, Instituto Nacional de Salud, Profamilia. Encuesta Nacional de la Situación Nutricional en Colombia 2010. 2011;1-512.

37. Ruiz ED, Arrubla DJ, Sanabria P. Envejecimiento y vejez en Colombia. 2013. [Internet]. [Consultado el 12 de Mayo 2015]. Disponible en: http://www.profamilia.org.co/docs/estudios/imagene s/3\%20\%20ENVEJECIMIENTO\%20Y\%20VEJEZ\%20EN\%20CO LOMBIA.pdf 